\title{
Incidental Discovery of Retained Triple Lumen Catheter Guidewire
}

\author{
Allen F. Shih, A.M $M^{7}$ and Christopher Sankey, M.D $D^{1,2}$ \\ ${ }^{1}$ Yale University School of Medicine, New Haven, CT, USA; ${ }^{2}$ Yale-New Haven Hospital, New Haven, CT, USA.
}

KEY WORDS: patient safety; medical error; clinical image.

J Gen Intern Med 32(3):361-2

DOI: $10.1007 / \mathrm{s} 11606-016-3798-8$

(c) Society of General Internal Medicine 2016

\section{DESCRIPTION}

A 30-year-old male with type 1 diabetes mellitus presented with diabetic ketoacidosis, acute kidney injury, and respiratory failure requiring emergent venous access and intubation. A left femoral triple lumen catheter (TLC) was inserted by an internal medicine resident with attending supervision. There were no reported complications. Fourteen days later, interventional radiology retrieved an "extra wire" via the right internal jugular vein at the time of hemodialysis catheter insertion. This

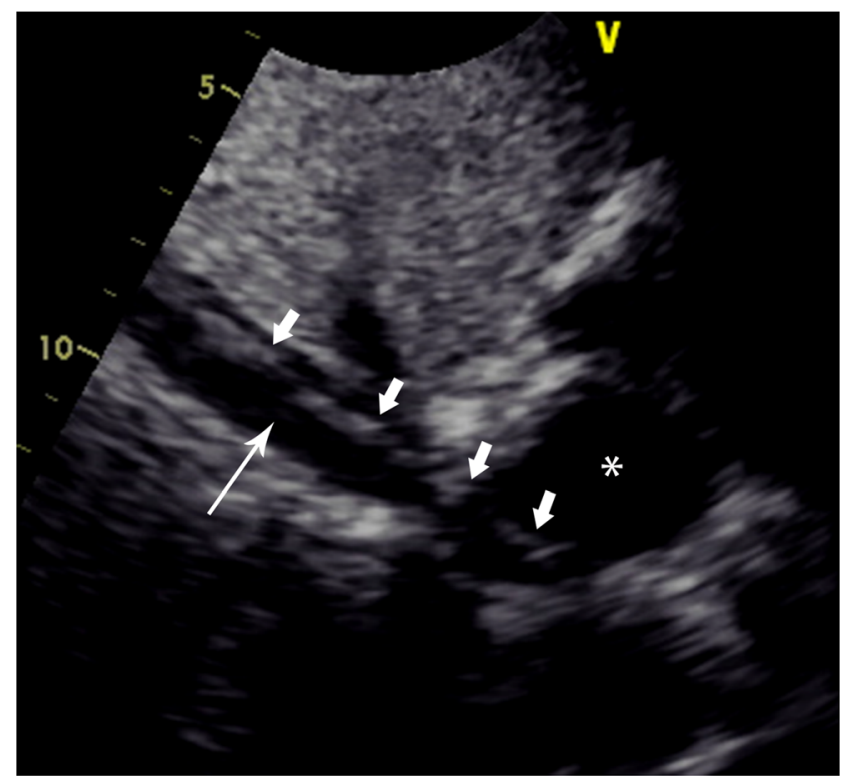

Figure 1. Transthoracic echocardiogram, demonstrating the guidewire (thick arrows) in the inferior vena cava (thin arrow) and right atrium (asterisk).

Electronic supplementary material The online version of this article (doi:10.1007/s11606-016-3798-8) contains supplementary material, which is available to authorized users.

Received February 29, 2016

Revised April 22, 2016

Accepted June 23, 2016

Published online July 11, 2016

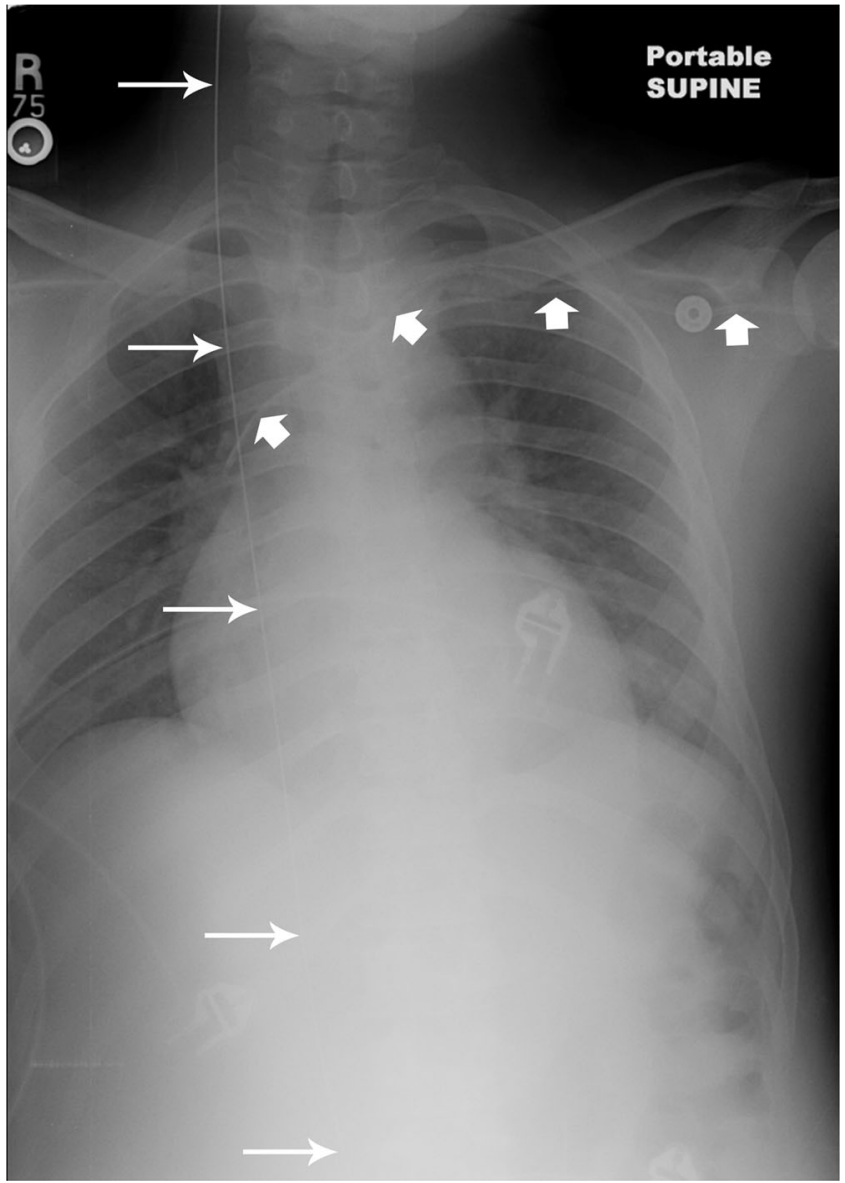

Figure 2. Chest X-ray, with the retained guidewire in the inferior and superior vena cava (thin arrows) and left subclavian intravascular catheter (thick arrows).

was determined to be the guidewire from the prior femoral TLC placement. On retrospective review of imaging, the guidewire can be seen on an echocardiogram (Fig. 1, online video) and multiple chest radiographs (Fig. 2) obtained between TLC insertion and guidewire retrieval. This "inattentional blindness," in which an unexpected visual finding is overlooked when focusing on a different task, was a substantial contributor to this medical error. ${ }^{1}$ The patient did not suffer any complications from the retained guidewire, and the error was disclosed to the patient. While more than 5 million central venous catheters are placed in the United States yearly, ${ }^{2}$ intravascular loss of complete guidewires represents a rare and serious complication that is preventable with standardized insertion technique and adequate trainee supervision. ${ }^{3}$ 
Corresponding Author: Allen F. Shih, A.M; Yale University School of Medicine, New Haven, CT, USA (e-mail: allen.shih@yale.edu).

\section{Compliance with Ethical Standards:}

Conflict of Interest: The authors declare that they do not have a conflict of interest.

\section{REFERENCES}

1. Drew T, Vo ML-H, Wolfe JM. The invisible gorilla strikes again: sustained inattentional blindness in expert observers. Psychol Sci. 2013;24(9):18481853. doi: $10.1177 / 0956797613479386$.

2. McGee DC, Gould MK. Preventing complications of central venous catheterization. N Engl J Med. 2003;348(12):1123-1133.

3. Schummer W. Loss of the guide wire: mishap or blunder? $\mathrm{Br} \mathrm{J}$ Anaesth. 2002;88(1):144-146. doi:10.1093/bja/88.1.144. 